\title{
A Novel Functional Swallow Method to Establish the Posterior Palatal Seal during the Maxillary Edentulous Final Impression: A Case Report
}

\author{
Ravishankar Krishna ${ }^{1}$, Rashmi B Mandokar ${ }^{2}$, Sweekriti Mishra ${ }^{3}$, Karishma Jagadeesh ${ }^{4}$, Suma Janya ${ }^{5}$, Babashankara Alva ${ }^{6}$
}

\begin{abstract}
Aim and objective: To propose an alternate, reliable, and easy-to-use functional swallow method for establishing the posterior palatal seal. Background: Accurate registration of the posterior palatal seal is crucial to the success of the maxillary complete denture. The conventionally used functional " $\mathrm{Ah}$ " technique may not be reliable at times to record the posterior palatal seal, hence compromising the retention of the maxillary complete denture.

Case description and technique: A 70-year-old female patient requiring complete dentures presented with an unusually tense soft palate during the phase of posterior palatal seal development. The soft palate did not relax and remained in a superior position when the "Ah" functional method was used to record the seal. The posterior palatal seal area could not be compressed sufficiently during border molding, preventing the formation of an adequate seal. Hence, a new functional technique was devised to relax and displace the soft palate for developing an effective seal. This case report describes a novel and reliable method of displacing the posterior palatal seal area with a low-fusing compound by utilizing the patient's functional swallow position.

Conclusion: The functional swallow method can be used effectively as an alternative and reliable method to obtain a consistent posterior palatal seal.

Clinical significance: The functional swallow method of developing the posterior palatal seal is simple, less demanding on the clinician, and can save chairside time. It also helps in an easy transfer of the demarcated posterior palatal seal area to the custom tray. The method lets the dentist be in charge of developing the seal rather than relying on the arbitrary cast scrapping by the technician. Also, the seal can be achieved by employing commonly used materials.

Keywords: Clinical technique, Complete dentures, Final impression, Functional swallow method, Maxillary complete denture, Maxillary denture retention, Posterior palatal seal.

The Journal of Contemporary Dental Practice (2020): 10.5005/jp-journals-10024-2988
\end{abstract}

\section{INTRODUCTION}

One of the primary reasons for lack of maxillary denture retention is a poor posterior palatal seal (PPS). ${ }^{1}$ Accurate registration of the PPS area plays a very critical role in the retention of maxillary complete denture. ${ }^{2}$ The commonly used approaches to develop the PPS can be classified into conventional, arbitrary, and functional methods. ${ }^{3}$ The conventional and arbitrary techniques are commonly taught and followed. ${ }^{4,5}$ Both the techniques involve scoring or scraping of the maxillary cast to establish the PPS. Scoring methods are not recommended as they are considered inaccurate and leave the retention of the denture to chance at the denture insertion appointment. ${ }^{6}$ The PPS area may also get over or undercompressed due to scoring and the seal achieved also may not be reliable.

Techniques that help to develop PPS during the border molding or final impression stage are favorable to the dentist. This is because the dentist becomes totally in charge of locating and developing the seal without relying on the technician. ${ }^{7}$ Development of PPS during the impression stage was classified by Hardy and Kapoor into functional and semi-functional methods. They explained that in the semi-functional technique, the molding of the seal area is done by the dentist whereas in the functional method, the molding of the PPS is performed by the patient's functional movements. ${ }^{8}$ Recently introduced semi-functional techniques to achieve the PPS have employed materials like light polymerized $\operatorname{resin}^{9}$ and specially formulated nonfluid waxes ${ }^{10}$ on the elastomeric final impression surface. The drawbacks of these methods can be the requirement

\footnotetext{
${ }^{1-6}$ Department of Prosthodontics, Faculty of Dental Sciences, Ramaiah University of Applied Sciences, Bengaluru, Karnataka, India

Corresponding Author: Ravishankar Krishna, Department Of Prosthodontics, Faculty of Dental Sciences, Ramaiah University of Applied Sciences, Bengaluru, Karnataka, India, Phone: +91 9886056303, e-mail: ravishankar.pr.ds@msruas.ac.in

How to cite this article: Krishna R, Mandokar RB, Mishra S, et al. A Novel Functional Swallow Method to Establish the Posterior Palatal Seal during the Maxillary Edentulous Final Impression: A Case Report. J Contemp Dent Pract 2020;21(12):1404-1407.

Source of support: Nil

Conflict of interest: None
}

of special waxes that need careful heating after its application on the final impression surface, ${ }^{10}$ extra caution during cast pouring to prevent wax distortion, ${ }^{7,10}$ and a possible overcompression of the PPS area due to the hard nature of resins. ${ }^{9}$

The functional method commonly used to achieve the PPS is through " $\mathrm{Ah}$ " sounds made by the patient to induce soft palate elevation during impression making. ${ }^{11}$ But at the completion of "Ah" sound, the soft palate returns early to its lower position before the modeling plastic impression compound may have hardened, compromising the PPS. ${ }^{9}$ Occasionally, in some patients the palatal 
muscles remain tensed and may not relax when asked to say " $\mathrm{Ah}$ " vigorously during the PPS formation procedure. In such patients, it may be difficult to displace the PPS area by the " $\mathrm{Ah}$ " functional method, and thereby an effective seal will not be formed. A novel functional technique utilizing the physiologic swallowing forces can form a consistent and successful PPS for these individuals when the head is flexed forward. So this case report aims to describe an alternate method to establish the PPS using the functional swallow position and low-fusing compound during the phase of complete denture final impression.

\section{TECHNIQUe}

\section{Background}

A 70-year-old completely edentulous female patient reported to the Department of Prosthodontics with a complaint of old and ill-fitting removable complete dentures. The patient was using these dentures for the past 6 years and requested a new pair, after considering the different treatment options given to her. On intraoral examination, moderately resorbed completely edentulous maxillary and mandibular ridges were seen. It was observed that the PPS area was of class II type based on House's classification of the palatal throat form. ${ }^{12}$ An informed consent was obtained from the patient and her relative before starting the treatment. Primary impressions of both arches were made in alginate and the casts were poured with plaster. Accurately fitting maxillary and mandibular resin custom trays were fabricated on the plaster casts. Border molding was performed using the polyvinyl siloxane (PVS) putty consistency impression material (elite HD+, Zhermack S.p.A. Italy) after applying a tray adhesive. During border molding with putty, the PPS formation was attempted using the conventional method, by making the patient say "Ah" in vigorous bursts and maintaining finger pressure on the posterior part of the custom tray. However, the obtained PPS was found to be inadequate when tested by applying tipping finger pressure on the inner side of the tray handle. The putty representing the PPS was removed and a second attempt to record the seal was made. This time, a low-fusing green stick compound was used instead of putty, and the patient was instructed to say " $A h$ " in vigorous bursts. The obtained PPS was still inadequate despite repeated attempts. It was also recognized that the patient had difficulty in repeatedly saying " $\mathrm{Ah}$ " in vigorous bursts as her palatal muscles were in tension and would rarely relax during the process. Hence, an alternate method to develop the PPS was planned by combining the patient's functional swallow with a forward head posture to relax the palatal muscles.

\section{Technique}

- The anterior and posterior vibrating lines were located intraorally by asking the patient to say "Ah" and marked with an indelible pencil.

- The maxillary border molded tray was placed intraorally to transfer the marked PPS area onto the PVS putty material. Transferring the mark onto the putty was easier compared to the resin tray. The tray was removed from the mouth and the transferred mark was highlighted with the indelible pencil (Fig. 1A).

- Putty extending beyond the transferred PPS mark was removed with a sharp B.P blade. Using a round tungsten carbide trimmer under slow speed, the putty representing the PPS was made rough and thinned down almost to the resin tray (Fig. 1B).

- The patient was trained to lean forward, bend the head down (Fig. 1C), and swallow, with the instruction to keep the tongue against the palate during the swallow. The angle between the Frankfort plane and the horizontal plane was at least $45^{\circ}$ when the head was bent forward.

- A stick of low-fusing compound (DPI Pinnacle Tracing Sticks, India) was softened using a flame and applied on the roughened polyvinyl siloxane PPS area. The added compound was heated uniformly using an alcohol torch, tempered in hot water, and placed in the patient's mouth. The tray was supported with fingers on either side and the patient was asked to bend forward
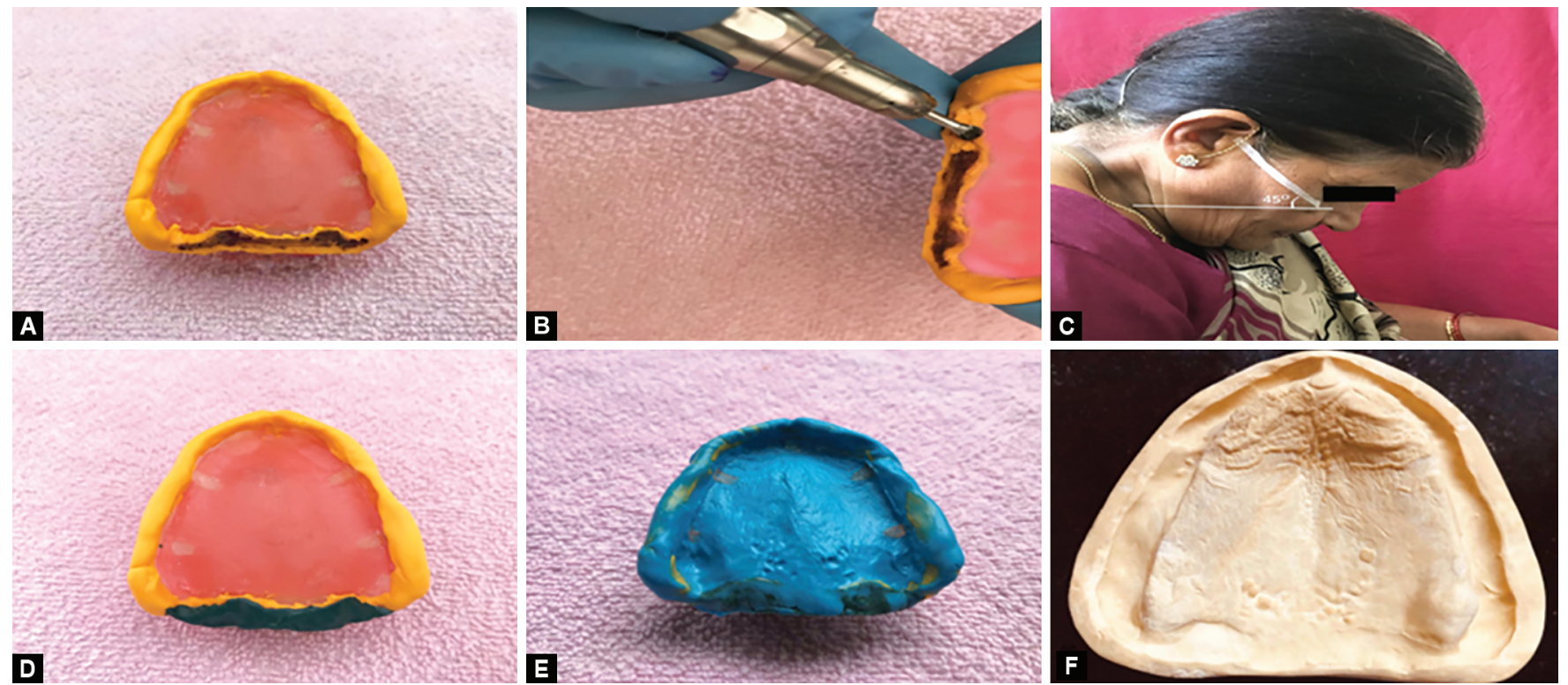

Figs 1 A to F: Steps to record the PPS with the novel functional swallow method: (A) Transfer of the PPS mark onto the elastomeric putty; (B) Roughening and thinning of the elastomeric putty representing the PPS; (C) Patient position for recording the PPS using the functional swallow method; (D) Low-fusing compound representing the PPS; (E) Low-viscosity elastomeric final impression; (F) Master cast with the functionally displaced PPS 
and swallow at least two times as practiced before. The patient was instructed to return to the normal posture and swallow once again. The efficacy of the PPS was assessed by tucking the tray handle on its inner side. A single application of the low-fusing compound was sufficient to achieve the desired seal. The green stick that appeared beyond the boundaries of the PPS was cut and finished using a sharp B.P blade (Fig. 1D).

- The final impression (Fig. 1E) was made using a light-body PVS impression material (elite HD+, Zhermack S.p.A. Italy). The impression was poured in type III gypsum to obtain a cast, with a custom-formed, functionally displaced PPS area (Fig. 1F).

\section{Prosthesis Outcome and Follow-Up}

The PPS developed using the novel swallow method gave increased confidence to the patient for wearing the maxillary complete denture. The patient was satisfied with the treatment outcome and could function adequately in speech and mastication. At 12-month follow-up, the patient presented with satisfactory retention and comfort of the maxillary complete denture. On clinical testing, the PPS was found to be consistent and similar to that developed during the final impression stage.

\section{Discussion}

Besides improving maxillary denture retention and stability, accurately formed PPS reduces gagging and makes the posterior border less noticeable to the tongue. It also adds strength to the denture and minimizes food accumulation on the underside. ${ }^{13-15}$ It partly compensates for the volumetric shrinkage of the acrylic resin and maintains intimate contact between the denture and tissues. ${ }^{16}$ The Glossary of Prosthodontic terms defines PPS area as "the soft tissue area limited posteriorly by the distal demarcation of the movable and non-movable tissues of the soft palate and anteriorly by the junction of the hard and soft palates on which pressure, within physiologic limits, can be placed; this seal can be applied by a removable complete denture to aid in its retention."17 This pressure within physiologic limits may probably be simulated from inherent functional processes that move the soft palate.

The commonly used functional method where the patient has to say "Ah" vigorously may be awkward and sometimes difficult to replicate. Also, the muscles of the soft palate are rather taut than relaxed during the pronunciation of "Ah" sounds. Hence, it may be difficult to displace the PPS area. Bending the head forward and downward can make the palatal muscles passive and relaxed. ${ }^{18}$ It is also known that the initial phase of swallowing function is voluntary, though swallowing reflex is considered involuntary. ${ }^{19}$ During this voluntary swallowing phase, the soft palate is elevated by the palatal muscles and the tongue comes in intimate contact with the palate, placing physiologic forces. ${ }^{20}$ This swallowing position of the soft palate and tongue, along with a forward-downward head posture can be used effectively by the dentist to displace the PPS area. The current technique incorporates this phenomenon and places functional swallowing pressure on the posterior part of the custom tray during PPS recording, which is inherent to the individual.

The PPS can be established at various stages of denture construction. It can be incorporated at the final impression stage $^{7}$ at the trial base stage ${ }^{15}$ or added to an existing denture. ${ }^{21}$ Developing the PPS during the final impression stage lets the dentist take responsibility for the process, as against the arbitrary and inaccurate cast scraping methods commonly used by technicians. ${ }^{22}$ The novel functional swallow technique described here helps in developing and estimating the PPS during the final impression phase. Today, elastomeric polyvinyl siloxane is commonly used as the final impression material for the construction of the tissuesupported removable prosthesis. ${ }^{5}$ Accordingly, the proposed functional swallow method was planned with elastomeric materials. Nevertheless, the novel technique is practical even with traditional border molding techniques that employ the low-fusing green stick compound.

The functional swallow method has the advantages of being simple, efficient, and consistent. Since the proposed technique does not rely on cast scoring for the development of PPS, it is less subjective and hence more consistent than the commonly followed cast scoring techniques. Elastomeric putty was used as the first layer during seal formation in the novel method. This helped in easy transfer of the demarcated PPS boundaries from the mouth onto the tray, which otherwise was difficult with the resin tray surface alone. The low-fusing compound (green stick) that is commonly used in practice was applied over the initial layer of putty in the present method to complete the seal. The advantage of using a low-fusing compound is its ease of addition and removal. Further, a low-fusing compound may not be undercompressive like the fluid waxes ${ }^{10,14}$ or overcompressive like resins. ${ }^{9}$ The viscosity of the lowfusing compound combined with the swallowing pressure was the reason for developing a consistent seal with the novel technique in the present clinical situation.

The fluid wax technique was developed by Millsap, ${ }^{14}$ whereas Wicks ${ }^{10}$ recently introduced the nonfluid wax technique for the development of PPS during the final impression. While fluid waxes are not dimensionally stable, nonfluid waxes contain sticky wax and hence may be difficult to soften after its application on the set elastomeric final impression surface. The proposed novel swallow method can be considered more accurate as it employs a lowfusing compound that is dimensionally stable when compared to waxes. A low-fusing compound (green stick) is easy to soften and is also more commonly used in practice when compared to fluid or nonfluid waxes. Also, additional precautions are not required to protect the PPS developed using a low-fusing compound during cast pouring, which is a necessity with waxes.

The resin-based provisional material was recently proposed by Batista ${ }^{23}$ whereas Jeannin ${ }^{9}$ advocated the light polymerized resin to be used on the elastomeric final impression surface to establish the PPS. Resin materials bond well to elastomeric surfaces but may be difficult to trim and finish especially when applied on an elastomeric final impression. Using methods that employ resin can be time-consuming, have the possibility of overcompression of the PPS area, and may lead to an eventual loss of the seal. ${ }^{23}$ The tendency for overcompression with the proposed swallow method is very less as the low-fusing compound is not as hard as the resins and also easy to trim.

Unlike the recently proposed methods, ${ }^{10,23}$ a major benefit of the novel method is that the PPS is incorporated before the light body final impression is made. This makes the technique less demanding when compared to contemporary techniques that require careful heating, cutting, or trimming of the PPS material applied onto the completed final impression surface to develop the posterior seal. Developing a post dam or PPS before the light 
body final impression can reduce the escape of excess material from the posterior tray end and hence prevent gagging. ${ }^{24}$ The disadvantages of the novel technique when compared with the recent techniques can include patient discomfort due to heat from the low-fusing compound and a possibility of an inadequate bond between the PVS putty impression material and the low-fusing compound. Tempering the compound close to mouth temperature can prevent patient discomfort. The inadequate bonding can be improved by creating roughness on putty with a carbide trimmer and also thinning down the putty to expose the resin tray in some areas so that the low fusing compound can bond with the tray. The other limitation of the swallow method can be inadequately formed PPS, especially if the tongue does not contact the palate during the swallow procedure. Proper communication and training the patient becomes important to prevent the said problem.

The purpose of describing this case report was to present a technique that the clinician can rely on and avoid cast scoring for developing the PPS. A study measured the maxillary denture retention of eight completely edentulous patients with their PPS established from a cast scraping method. The study involved follow-ups to check the retention at various stages that were evaluated by three clinicians. The research concluded that the scraping method used to establish the PPS was safe and effective. ${ }^{25}$ But, the study results may not be generalizable as the sample size was small and the retention was measured by subjective means. Hence, a well-designed study comparing the effectiveness of the conventional cast scoring method and the novel functional swallow method in achieving maxillary denture retention would benefit the clinician. Devices like the dynamometer may help in measuring and comparing the retention values objectively. ${ }^{26}$ Although, in the present case a reliable seal was established, the PPS anatomy can be variable and challenging especially in House's class III type of soft palates. In these patients, the compressible tissues are scanty to develop an effective seal. Further research is needed to prove the consistency of the novel functional swallow technique in similar demanding circumstances.

\section{ConcLusion}

Establishing accurate and efficient PPS in a maxillary complete denture is crucial for its success. Incorporating PPS during the final impression stage may have many advantages. The functional swallow technique described in this article was efficient and consistent for developing the PPS. Materials commonly used in practice were employed for developing the PPS. Most importantly, the displacement of the soft palate was brought about by functional swallowing forces that are inherent to an individual. The functional swallow method can be a reliable alternative for the development of PPS.

\section{References}

1. Winland RD, Young JM. Maxillary complete denture posterior palatal seal: variations in size, shape, and location. J Prosthet Dent 1973;29(3):256-261. DOI: 10.1016/0022-3913(73)90004-8.

2. Avant WE. A comparison of the retention of complete denture bases having different types of posterior palatal seal. J Prosthet Dent 1973;29(5):484-493. DOI: 10.1016/0022-3913(73)90025-5.

3. Goyal S. The posterior palatal seal: its rationale and importance: an overview. Eur J Prosthodont 2014;2(2):41-47. DOI: 10.4103/23474610.131972 .
4. Chen MS, Welker WA, Pulskamp FE, et al. Methods taught in dental schools for determining the posterior palatal seal region. J Prosthet Dent 1985;53(3):380-383. DOI: 10.1016/0022-3913(85)90517-7.

5. Petrie CS, Walker MP, Williams K. A survey of U.S. prosthodontists and dental schools on the current materials and methods for final impressions for complete denture prosthodontics. J Prosthodont 2005;14(4):253-262. DOI: 10.1111/j.1532-849X.2005.00051.x.

6. Winkler S. Essentials of complete denture prosthodontics. 3rd ed., New Delhi: AITBS Publishers; 2015. p. 160.

7. Ansari IH. Establishing the posterior palatal seal during the final impression stage. J Prosthet Dent 1997;78(3):324-326. DOI: 10.1016/ s0022-3913(97)70034-9.

8. Hardy IR, Kapur KK. Posterior border seal: its rationale and importance. J Prosthet Dent 1958;8(3):386-397. DOI: 10.1016/0022-3913(58)900647.

9. Jeannin C, Millet C. A functional impression technique for capturing the superior position of the soft palate. J Prosthet Dent 2006;96(2):145-146. DOI: 10.1016/j.prosdent.2006.05.014.

10. Wicks $R$, Ahuja $S$, Jain V. Defining the posterior palatal seal on a definitive impression for a maxillary complete denture by using a nonfluid wax addition technique. J Prosthet Dent 2014;112(6):15971600. DOI: 10.1016/j.prosdent.2014.01.032.

11. Hayakawa I. Principles and practices of complete dentures. Tokyo: Quintessence; 2001. p. 37.

12. House MM. The relationship of oral examination to dental diagnosis. J Prosthet Dent 1958;8(2):208-219. DOI: 10.1016/0022-3913(58)90150-1.

13. Ettinger RL, Scandrett FR. The posterior palatal seal. A review. Aust Dent J 1980;25(4):197-200. DOI: 10.1111/j.1834-7819.1980.tb03863.x.

14. Millsap $\mathrm{CH}$. The posterior palatal seal area for complete dentures. Dent Clin North Am 1964;1:663-673.

15. Chang BM, Wright RF. Accurate location of postpalatal seal area on the maxillary complete denture cast. J Prosthet Dent 2006;96(6):454-455. DOI: 10.1016/j.prosdent.2006.10.010.

16. Sykora O, Sutow EJ. Posterior palatal seal adaptation: influence of processing technique, palate shape and immersion. J Oral Rehabil 1993;20(1):19-31. DOI: 10.1111/j.1365-2842.1993.tb01511.x.

17. The glossary of prosthodontic terms: ninth edition. J Prosthet Dent 2017;117(5S):e1-e105. DOI: 10.1016/j.prosdent.2016.12.001.

18. Winkler S. Essentials of complete denture prosthodontics. 3rd ed., New Delhi: AITBS Publishers; 2015. p. 158.

19. Walton J, Silva P. Physiology of swallowing. Basic Science 2018;36(10):529-534. DOl: https://doi.org/10.1016/j. mpsur.2018.08.010.

20. Matsuo K, Palmer JB. Anatomy and physiology of feeding and swallowing: normal and abnormal. Phys Med Rehabil Clin N Am 2008;19(4):691-707. , vii 10.1016/j.pmr.2008.06.001.

21. Carroll EA, Shaffer FW. Redefining the posterior palatal seal on a complete denture. J Prosthet Dent 1980;43(1):105-107. DOI: 10.1016/0022-3913(80)90363-7.

22. Porter RB. Evaluation of a delegated procedure: posterior border of a maxillary denture. J Am Dent Assoc 1970;81(1):134-136. DOI: 10.14219/ jada.archive.1970.0150.

23. De Souza Batista VE, Vechiato-Filho AJ, Pellizzer EP, et al. Use of resin-based provisional material to create the posterior palatal seal in complete denture definitive impressions. J Prosthodont 2019;28(1):e18-e20. DOI: 10.1111/jopr.12658.

24. Weintraub GS. Establishing the posterior palatal seal during the final impression procedure: a functional approach. J Am Dent Assoc 1977;94(3):505-510. DOI: 10.14219/jada.archive.1977.0007.

25. Salloum AM. Evaluation of the conventional method for establishing the posterior palatal seal. King Saud Univ J Dent Sci 2012;3(2):61-67. DOI: 10.1016/j.ksujds.2012.07.001.

26. Jozefowicz W, Mikolajczyk A. Role of the surface and volume of palatal relief chambers in the retention of maxillary complete dentures. J Prosthet Dent 1989;62(6):651-653. DOI: 10.1016/0022-3913(89) 90585-4. 